\title{
Deep multi-frequency radio imaging in the Lockman Hole using the GMRT and VLA
}

\author{
Edo lbar* ${ }^{\dagger}$ \\ UK Astronomy Technology Centre, Royal Observatory Edinburgh \\ E-mail: ibareroe.ac.uk

\section{Rob Ivison} \\ UK Astronomy Technology Centre, Royal Observatory Edinburgh \\ Institute for Astronomy, University of Edinburgh \\ E-mail: rjieroe.ac.uk
}

\begin{abstract}
In the run up to routine observations with the upcoming generation of radio facilities, the nature of sub-mJy radio population has been hotly debated. In this talk, we describe multi-frequency radio data designed to probe the emission mechanism that dominates in these faint radio sources. Our analysis is based on observations of the Lockman Hole using the Giant Metre-wave Radio Telescope (GMRT) - the deepest 610-MHz imaging yet reported - together with 1.4-GHz imaging from the Very Large Array (VLA), well matched in resolution and sensitivity to the GMRT data $\left(\sigma_{610 \mathrm{MHz}} \sim 15 \mu \mathrm{Jy} / \mathrm{beam}, \sigma_{1.4 \mathrm{GHz}} \sim 6 \mu \mathrm{Jy} /\right.$ beam, FWHM $\left.\sim 5 \mathrm{arcsec}\right)$. The GMRT and VLA data are cross-matched to obtain the radio spectral indices for the faint radio emitters. Statistical analyses show no clear evolution for the median spectral index, $\alpha_{610 \mathrm{MHz}}^{1.4 \mathrm{GHz}}$ (where $S_{v} \propto v^{\alpha}$ ), which is found to be approximately -0.6 to -0.7 based on an almost unbiased 10-sigma criterion, down to a flux level of $S_{1.4 \mathrm{GHz}} \geq 100 \mu \mathrm{Jy}$. The fraction of inverted spectrum sources, $\alpha_{610 \mathrm{MHz}}^{1.4 \mathrm{GHz}} \geq 0$, is less than $10 \%$. The results suggest that the most prevalent emission mechanism in the sub-mJy regime is optically-thin synchrotron, ruling out a dominant flat spectrum or an aged ultra-steep spectrum radio population. The spectral index distribution has a significant scatter, $\Delta \alpha \sim 0.4-0.5$, suggesting a mixture of different populations at all flux levels. This is supported by spectroscopic classifications of radio sources with X-ray emission, which has allowed us to estimate that the fraction of radio-quiet (type 1 and 2) active galactic nuclei (AGN) at $30 \mu \mathrm{Jy}<\mathrm{S}_{1.4 \mathrm{GHz}} \leq 300 \mu \mathrm{Jy}$ is $15-35 \%$, consistent with a dominant star-forming galaxy population in the sub-mJy regime.
\end{abstract}

Panoramic Radio Astronomy: Wide-field 1-2-GHz research on galaxy evolution - PRA2009

June 02-05 2009

Groningen, the Netherlands

\footnotetext{
* Speaker.

${ }^{\dagger}$ This talk is based on the publication by Ibar et al. (2009).
} 


\section{Introduction}

In early studies, radio astronomy was limited to bright sources associated with rare luminous radio galaxies and quasars (QSOs), usually hosted by massive elliptical galaxies [14]. It was not until the mid-1980s that radio telescopes started to shed light on objects with sub-mJy levels, revealing a new population - usually bluer and associated with star-forming galaxies instead of AGN (e.g. [21]). Nowadays, the improved sensitivity reached by radio facilities has allowed the detection of a large number of faint sub-mJy radio sources, although the fraction of AGN and star-forming galaxies that contribute to this population remains largely unknown. The nature of this faint population is being hotly debated, e.g. [1], 18].

In this talk, we present a study of these sources, looking at their radio spectral properties. We have combined radio data at frequencies where synchrotron emission is the dominant emission process. We merge two datasets to characterise the spectral index of the $\mu \mathrm{Jy}$ radio population as a function of flux density, thereby probing the physical mechanisms that dominate in this enigmatic population: optically thin (steep spectrum) or self-absorbed (hard spectrum) synchrotron emission. We have also analysed their X-ray properties in order to quantify the fraction of AGNs in the sample.

\section{The sub-mJy radio population}

We obtained two very deep radio images centred on the Lockman Hole (LH): the deepest to date at $610 \mathrm{MHz}\left(\sigma \sim 15 \mu \mathrm{Jy} \mathrm{beam}^{-1}\right.$; FWHM $\left.=7.1 \times 6.5 \mathrm{arcsec}^{2}\right)$ using the GMRT and a deep 1.4GHz image $\left(\sigma \sim 6 \mu \mathrm{Jy}_{\text {beam }}{ }^{-1}\right.$; FWHM $\left.=4.3 \times 4.2 \operatorname{arcsec}^{2}\right)$ using the VLA. In a total area of 3,534 and 2,019 $\operatorname{arcmin}^{2}$, we have found 1,587 and 1,452 sources $(>5-\sigma)$, at $610 \mathrm{MHz}$ and $1.4 \mathrm{GHz}$, respectively.
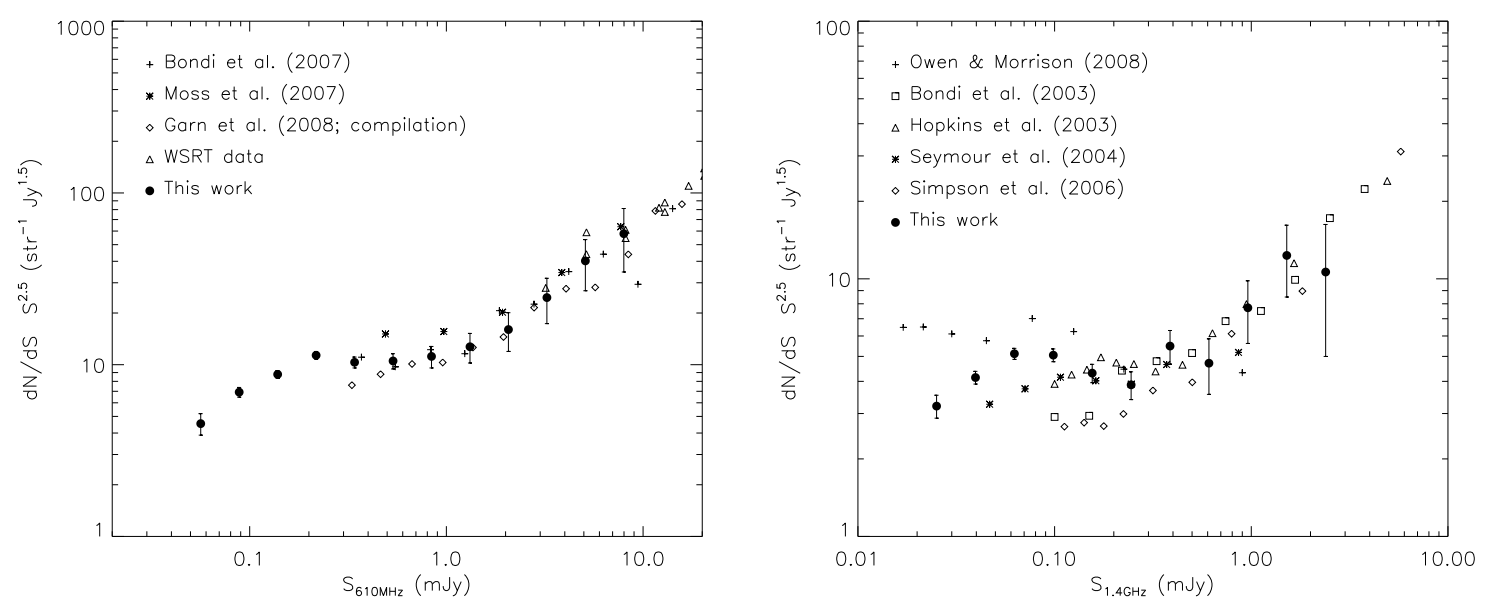

Figure 1: Euclidean number counts as a function of flux density in the Lockman Hole at $610 \mathrm{MHz}(l e f t)$ and $1.4 \mathrm{GHz}$ (right). Errors are assumed to be Poissonian in counts. At $610 \mathrm{MHz}$, and 1.4-GHz we compare our results with previous surveys. 
Based on the large number of sources, we have estimated the differential number counts down to $\mu \mathrm{Jy}$ flux densities (see Fig. 11). We observe a flattening in the Euclidean differential number counts towards sub-mJy flux densities at both $610 \mathrm{MHz}$ and $1.4 \mathrm{GHz}$. We find evidence for a second peak in number counts at $\sim 80 \mu \mathrm{Jy}$ and $\sim 200 \mu \mathrm{Jy}$ for the VLA and GMRT counts, respectively.

Our number counts are in good agreement with previous bright $(>100 \mu \mathrm{Jy})$ surveys, although a large scatter is seen at fainter 1.4-GHz flux densities. This may be due to underestimation of the number counts in shallow images; for example, Owen et al. [16] reported an approximately flat $\log N-\log S$ distribution down to $S_{1.4 \mathrm{GHz}} \sim 15 \mu \mathrm{Jy}$, exploiting an extremely deep VLA 1.4-GHz image of The Deep Swire Field $(\sigma \sim 3 \mu \mathrm{Jy})$. The origin of the wide scatter in reported 1.4-GHz number counts is controversial. It is possible that for $<500 \mu \mathrm{Jy}$ sources our survey area is not large enough to average out cosmic structure [7] - note that for a field subtending an angle of one square degree, the angular diameter distance at redshift unity is only $\sim 20 \mathrm{Mpc}$. On the other hand, noise fluctuations can contaminate the number counts through the inclusion of spurious sources and by boosting intrinsically faint sources to higher flux densities. After a detailed analysis, we concluded that most of the scatter at faint flux densities is due to the different corrections applied to the number counts due to: the non-homogeneous noise across the image, the efficiency of the source extraction method and the uncertainties in the intrinsic source size distribution.

\section{Radio spectral indices}

We have cross-matched (within 7 arcsec) the GMRT and VLA radio catalogues in order to estimate the spectral index of the radio emitters. The distribution of $\alpha_{1.4 \mathrm{GHz}}^{610 \mathrm{MHz}}$ shows a large scatter $\sigma_{\alpha} \approx 0.4$ in the sub-mJy regime (Fig. 2, left), which suggests the detection of a variety of different populations - normal star-forming galaxies, probably a substantial number of synchrotron selfabsorbed AGN cores [2] and high-redshift ultra-steep-spectrum (USS) sources [11] - casting doubt on previous studies which assume a clean star-forming galaxy population with a single spectral index in the sub-mJy radio regime. Roughly $6 \%(\lesssim 10 \%$ - upper limit) of the sources have $\alpha>0$ in the catalogues - these sources look compact at both wavelengths.

In terms of median and mean values (Fig. 2, right), roughly within -0.7 and -0.6 (similar to that found in local star-forming galaxies [6]), we find no clear trend in the spectral indices toward fainter flux densities in either the GMRT- or the VLA-selected samples. Since these two samples tend to select spectra with different spectral indexes (flatter and steeper for higher and lower frequencies, respectively), numerical differences of $\sim 0.2$ in the mean $\alpha$, and $\sim 0.1$ in the median, are seen in the estimates for similar flux density bins. These variations reflect the nonGaussianity and the large scatter of the distribution, especially at the faintest detections.

We suggest the sub-mJy radio population is dominated by optically-thin synchrotron emission from star-forming galaxies and/or from steep-spectrum lobe-dominated Fanaroff-Riley (FR) AGN [8]. These results contradict the suggested flattening in spectral index at sub-mJy radio flux densities quoted in previous studies (see Table 1).

\section{X-ray identifications}

To identify AGN sources in the sub-mJy radio population we have cross-matched our VLA 

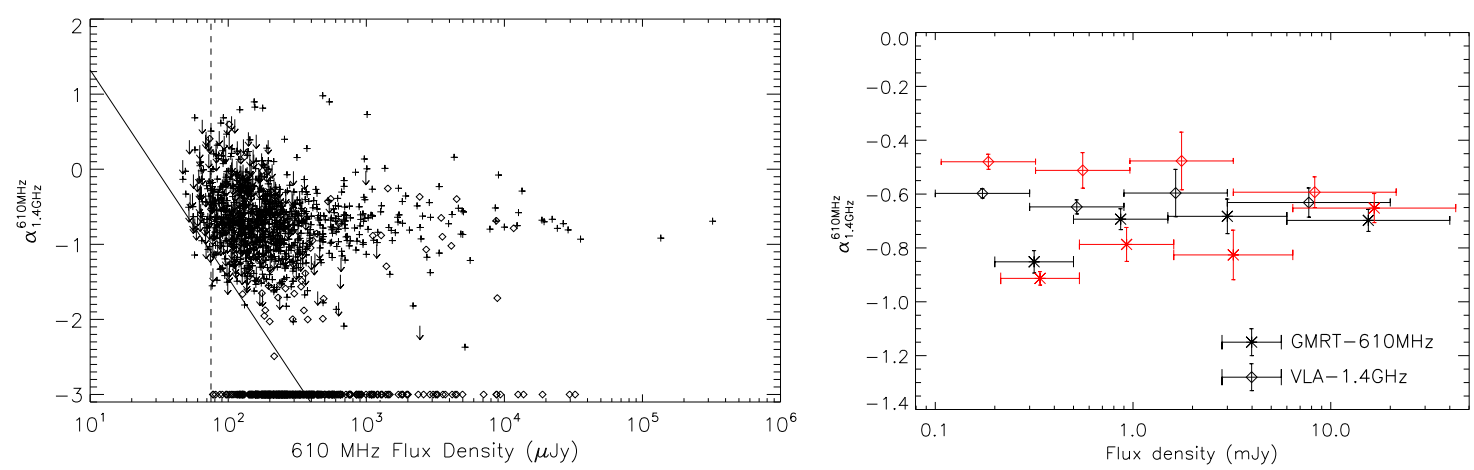

Figure 2: The radio spectral indices as a function of flux density based on a 610-MHz-selected sample (left). Plus symbols are detections (limited to $10-$ and $5-\sigma$ at $610 \mathrm{MHz}$ and $1.4 \mathrm{GHz}$, respectively). Dashed and solid lines show the flux limits for each survey. Arrows are 5- $\sigma$ upper limits. Diamonds show unreliable spectral indexes. Data at $\alpha=-3$ are sources outside the overlapping region. On the right, the medianbootstrap (in black) and the Kaplan-Meier mean (in red) spectral indices as a function of flux density. Data are based on 610-MHz- and 1.4-GHz-selected samples. In the image, mean values are slightly shifted in flux density (just for clarity).

\begin{tabular}{|cccc|}
\hline Reference & Flux range $(\mathrm{mJy})$ & $\left\langle\left\langle\alpha_{1.4 \mathrm{GHz}}^{610 \mathrm{MHz}}\right\rangle\right\rangle_{\text {median }}^{\mathrm{Ref}}$ & $\left\langle\left\langle\alpha_{1.4 \mathrm{GHz}}^{610 \mathrm{MHz}}\right\rangle\right\rangle_{\text {median }}^{\text {Ours }}$ \\
\hline Bondi et al. [3] & $0.5<S_{1.4 \mathrm{GHz}}$ & $-0.67 \pm 0.05$ & $-0.63 \pm 0.03$ \\
& $0.15<S_{1.4 \mathrm{GHz}}<0.5$ & $-0.46 \pm 0.03$ & $-0.63 \pm 0.02$ \\
& $0.1<S_{1.4 \mathrm{GHz}}<0.15$ & $-0.61 \pm 0.04$ & $-0.59 \pm 0.03$ \\
\hline Garn et al. [6] & $1.0<S_{610 \mathrm{MHz}}$ & $-0.56 \pm 0.04$ & $-0.69 \pm 0.04$ \\
& $0.5<S_{610 \mathrm{MHz}}<1.0$ & $-0.36 \pm 0.12$ & $-0.71 \pm 0.04$ \\
\hline Tasse et al. [19] & $30<S_{610 \mathrm{MHz}}$ & -0.76 & $-0.71 \pm 0.17$ \\
\hline
\end{tabular}

Table 1: A direct comparison of our results with previous work.

1.4-GHz catalogue with the deepest XMM/Newton catalogue so far published [5]. In Fig. 3 (left) we show the fraction of radio sources detected in the hard X-ray band - a good indicator of AGN activity - as a function of radio flux density. We find that this fraction declines from $\sim 30 \%$ at $\sim 1 \mathrm{mJy}$ to $\sim 10 \%$ at $\lesssim 100 \mu \mathrm{Jy}$. This suggests that the fraction of AGN decreases as we go to fainter sub-mJy flux densities.

We use the optical spectroscopic classification presented by Brunner et al. [5] to plot the hard $\mathrm{X}$-ray and 1.4-GHz flux densities (Fig. 3, right) as a function of different populations. We find that in the sub-mJy radio regime, the number of normal galaxies at fainter $\left(S_{2-10 \mathrm{keV}} \lesssim 3 \times 10^{-15}\right.$ erg $\sec ^{-1} \mathrm{~cm}^{-2}$ ) X-ray flux densities increases, therefore a hard X-ray counterpart does not ensure an AGN nature. Taking into account the spectroscopic classification, we have considered a rough estimation for the total fraction of radio-quiet AGN in the sub-mJy radio regime based on four assumptions: (1) the X-ray catalogue contains almost all the type-1 AGN in the redshift range of the radio sources (mean, $z \approx 0.8$ ); (2) to estimate an upper limit the spectroscopically-identified sources maintain the same relative fractions in the unknown population as in Fig. 3 (right); (3) a constant fraction of type 1/type 2 AGN $=1: 4$ based on X-ray observations of local Seyfert galaxies, e.g. [13]; (4) $25 \%$ of the X-ray sources are Compton-thick (undetected) based on synthesis population 

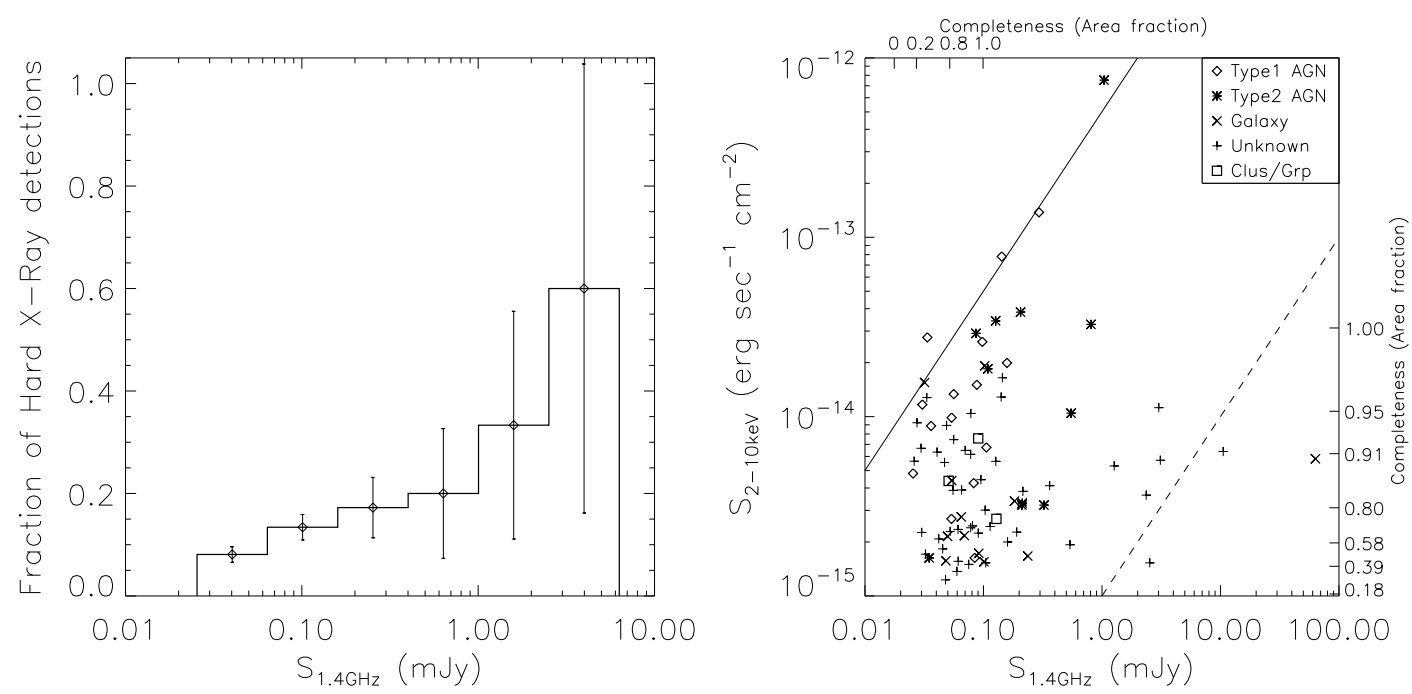

Figure 3: Left: The fraction of radio sources (at $1.4 \mathrm{GHz}$ ) detected by XMM/Newton in the hard X-ray band. Errors are assumed to be Poissonian in counts. Right: Radio flux density versus X-ray flux density for cross-matched sources. The solid and dashed lines correspond to the expected correlation for radio-quiet AGN [4] and star-forming galaxies [6, 17] introduced by Simpson et al. [18], respectively. The different populations are based on spectroscopic identifications ([12] and Szokoly et al., in preparation) compiled by Brunner et al. [5].

models to reproduce the Cosmic X-ray Background, e.g. [20]. Under these assumptions, we expect an AGN contribution of $15-35 \%$ in the $30 \mu \mathrm{Jy} \leq S_{1.4 \mathrm{GHz}}<300 \mu \mathrm{Jy}$ radio population. We conclude at sub-mJy radio flux levels a transition from a bright and powerful AGN to a dominant star-forming galaxy population, contaminated at the $\sim 15-35 \%$ level by radio-quiet AGN at $S_{1.4 \mathrm{GHz}} \approx 100 \mu \mathrm{Jy}$.

\section{Concluding remarks}

We have observed the Lockman Hole field using the GMRT at $610 \mathrm{MHz}$ and the VLA at $1.4 \mathrm{GHz}$, obtaining two deep radio images with similar spatial resolutions and well-matched noise levels ( 15 and $6 \mu \mathrm{Jy}_{\text {beam }}{ }^{-1}$, respectively) - the former representing the deepest GMRT image yet published. The data reveal a flattening followed by a second peak in the Euclidean-normalised number counts in the sub-mJy radio regime - evidence for the appearance of a different dominant radio population at these faint flux densities.

We study the spectral index of the radio emitters by combining our deep, well-matched observations - about three times deeper than previous data - which mitigate the well-known bias towards the detection of steeper spectra at longer wavelengths, or flatter spectra at shorter wavelengths. Our results, show that the mean and median spectral index does not evolve strongly as a function of radio flux density, certainly between $\sim 100 \mu \mathrm{Jy}$ and $10 \mathrm{mJy}$ at $1.4 \mathrm{GHz}$. We find $\alpha_{1.4 \mathrm{GHz}}^{610 \mathrm{MHz}} \approx-0.6$ to -0.7 , which suggests that optically-thin synchrotron emission is the dominant emission mechanism in the sub-mJy population - similar to local star-forming galaxies. These findings rule out a possible dominant flat-spectrum population (e.g. AGN-cores; GPS) at these faint flux densities 
[3, 9]. The distribution of spectral indices has a significant scatter $\left(\sigma_{\alpha} \approx 0.4\right)$ suggesting a complicated scenario where different populations mix together.

Based on X-ray observations with spectroscopic classifications [5], we estimate that approximately $\sim 15-35 \%$ of the radio sample is made up of radio-quiet AGN at $30 \mu \mathrm{Jy} \lesssim S_{1.4 \mathrm{GHz}}<300 \mu \mathrm{Jy}$, with this fraction rising towards brighter flux densities. These results suggest a transition from AGN to a dominant star-forming population at sub-mJy radio fluxes.

\section{References}

[1] Barger A. J., Cowie L. L., Wang W.-H., 2007, ApJ, 654, 764

[2] Blundell K. M., Kuncic Z., 2007, ApJ, 668, L103

[3] Bondi M. et al., 2007, A\&A, 463, 519

[4] Brinkmann W., Laurent-Muehleisen S. A., Voges W., Siebert J., Becker R. H., Brotherton M. S., White R. L., Gregg M. D., 2000, A\&A, 356, 445

[5] Brunner H., Cappelluti N., Hasinger G., Barcons X., Fabian A. C., Mainieri V., Szokoly G., 2008, A\&A, 479, 283

[6] Condon J. J., 1992, ARA\&A, 30, 575

[7] Condon J. J., 2007, in Astronomical Society of the Pacific Conference Series, Vol. 380, Afonso J., Ferguson H. C., Mobasher B., Norris R., eds, Deepest Astronomical Surveys, p. 189

[8] Fanaroff B. L., Riley J. M., 1974, MNRAS, 167, 31P

[9] Garn T., Green D. A., Riley J. M., Alexander P., 2008a, MNRAS, 383, 75

[10] Garn T., Green D. A., Riley J. M., Alexander P., 2008b, MNRAS, 387, 1037

[11] Jarvis M. J. et al., 2001, MNRAS, 326, 1563

[12] Lehmann I. et al., 2001, A\&A, 371, 833

[13] Maiolino R., Salvati M., Bassani L., Dadina M., della Ceca R., Matt G., Risaliti G., Zamorani G., 1998, A\&A, 338, 781

[14] Matthews T. A., Morgan W. W., Schmidt M., 1964, ApJ, 140, 35

[15] Moss D., Seymour N., McHardy I. M., Dwelly T., Page M. J., Loaring N. S., 2007, MNRAS, 378, 995

[16] Owen F. N., Morrison G. E., 2008, AJ, 136, 1889

[17] Ranalli P., Comastri A., Setti G., 2003, A\&A, 399, 39

[18] Simpson C. et al., 2006, MNRAS, 372, 741

[19] Tasse C., Röttgering H. J. A., Best P. N., Cohen A. S., Pierre M., Wilman R., 2007, A\&A, 471, 1105

[20] Ueda Y., Akiyama M., Ohta K., Miyaji T., 2003, ApJ, 598, 886

[21] Windhorst R. A., Miley G. K., Owen F. N., Kron R. G., Koo D. C., 1985, ApJ, 289, 494 